\title{
Identification of a novel metabolism-related prognostic signature in hepatocellular carcinoma through bioinformatics analysis and validation through experimental studies
}

\section{Zhihao Wang}

Huazhong University of Science and Technology Tongji Medical College

Kidane Siele Embaye

Huazhong University of Science and Technology Tongji Medical College

\section{Qing Yang}

Department of Pharmacy, Hiser Medical Center of Qingdao

\section{Lingzhi Qin Qin}

Huazhong University of Science and Technology Tongji Medical College

Chao Zhang

Huazhong University of Science and Technology Tongji Medical College

Liwei Liu

Huazhong University of Science and Technology Tongji Medical College

\section{Xiaoqian Zhan}

Huazhong University of Science and Technology Tongji Medical College

\section{Fengdi Zhang}

Department of Pathology, Wuhan Third Hospital (Tongren Hospital of Wuhan University )

\section{Xi Wang}

Huazhong University of Science and Technology Tongji Medical College

Shenghui Qin ( 2015 tj0147@ @hust.edu.cn )

Huazhong University of Science and Technology Tongji Medical College https://orcid.org/0000-00020275-133X

\section{Research}

Keywords: Hepatocellular carcinoma, Metabolism-related genes, Prognostic signature, Survival

Posted Date: November 13th, 2020

DOl: https://doi.org/10.21203/rs.3.rs-104555/v1 
License: (c) (i) This work is licensed under a Creative Commons Attribution 4.0 International License. Read Full License 


\section{Abstract}

\section{Background}

Given that metabolic reprogramming has been recognized as an essential hallmark of cancer cells, this study sought to investigate the potential prognostic values of metabolism-related genes(MRGs) for hepatocellular carcinoma (HCC) diagnosis and treatment.

\section{Methods}

The metabolism-related genes sequencing data of HCC samples with clinical information were obtained from the International Cancer Genome Consortium(ICGC) and The Cancer Genome Atlas (TCGA). The differentially expressed MRGs were identified by Wilcoxon rank sum test. Then, univariate Cox regression analysis were performed to identify metabolism-related DEGs that related to overall survival(OS) . A novel metabolism-related prognostic signature was developed using the least absolute shrinkage and selection operator (Lasso) and multivariate Cox regression analyses . Furthermore, the signature was validated in the TCGA dataset. Finally, the expression levels of hub genes were validated in cell lines by Western blotting (WB) and quantitative real-timePCR (qRT-PCR).

Results

A total of 178 differentially expressed MRGs were detected between the ICGA dataset and the TCGA dataset. We found that 17 MRGs were most significantly associated with OS by using the univariate Cox proportional hazards regression analysis in HCC. Then, the Lasso and multivariate Cox regression analyses were applied to construct the novel metabolism-relevant prognostic signature, which consisted of six MRGs. The prognostic value of this prognostic model was further successfully validated in the TCGA dataset. Further analysis indicated that this signature could be an independent prognostic indicator after adjusting to other clinical factors. Six MRGs (FLVCR1, MOGAT2, SLC5A11, RRM2, COX7B2, and SCN4A) showed high prognostic performance in predicting HCC outcomes. Finally, hub genes were chosen for validation and the expression of FLVCR1, SLC5A11, and RRM2 were significantly increased in human hepatocellular carcinoma cell lines when compared to normal human hepatic cell line, which were in agreement with the results of differential expression analysis.

\section{Conclusions}

In summary, our data provided evidence that the metabolism-based signature could serve as a reliable prognostic and predictive tool for overall survival in patients with HCC.

\section{Full Text}

This preprint is available for download as a PDF.

\section{Figures}


Fig.1

a

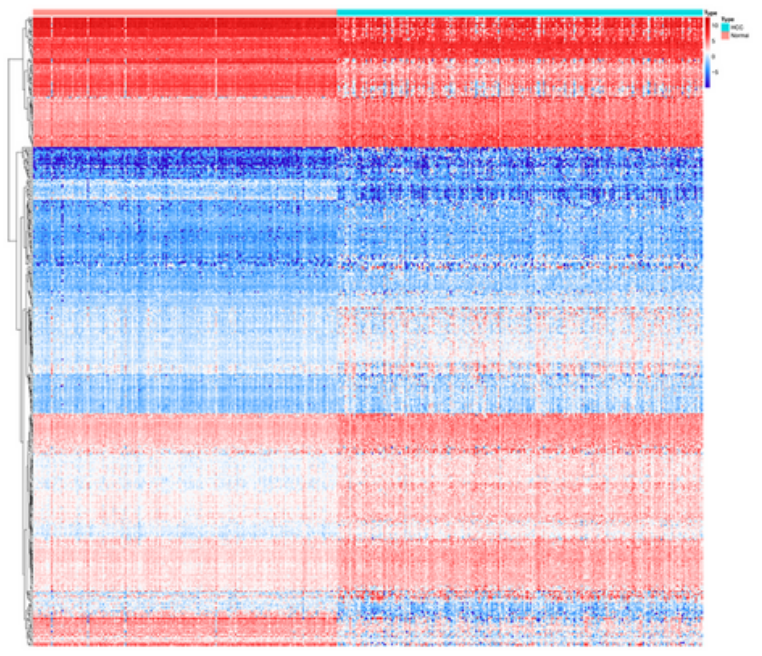

b

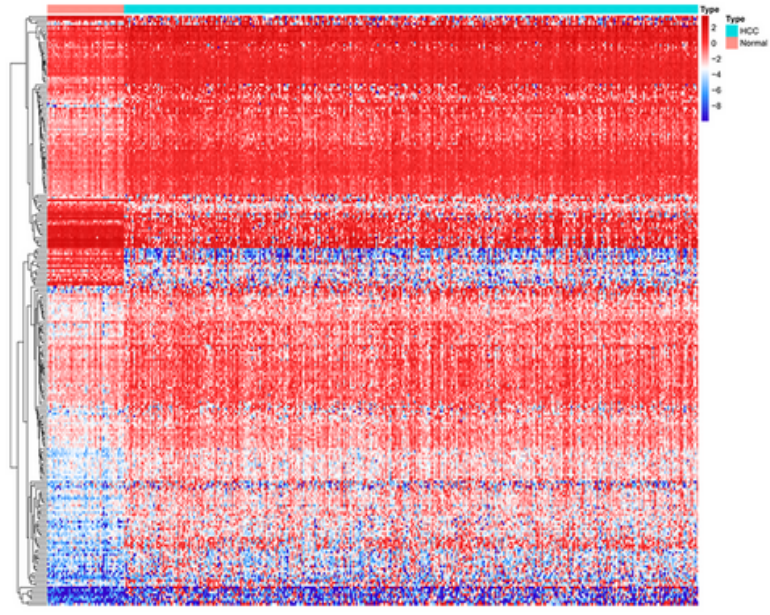

e
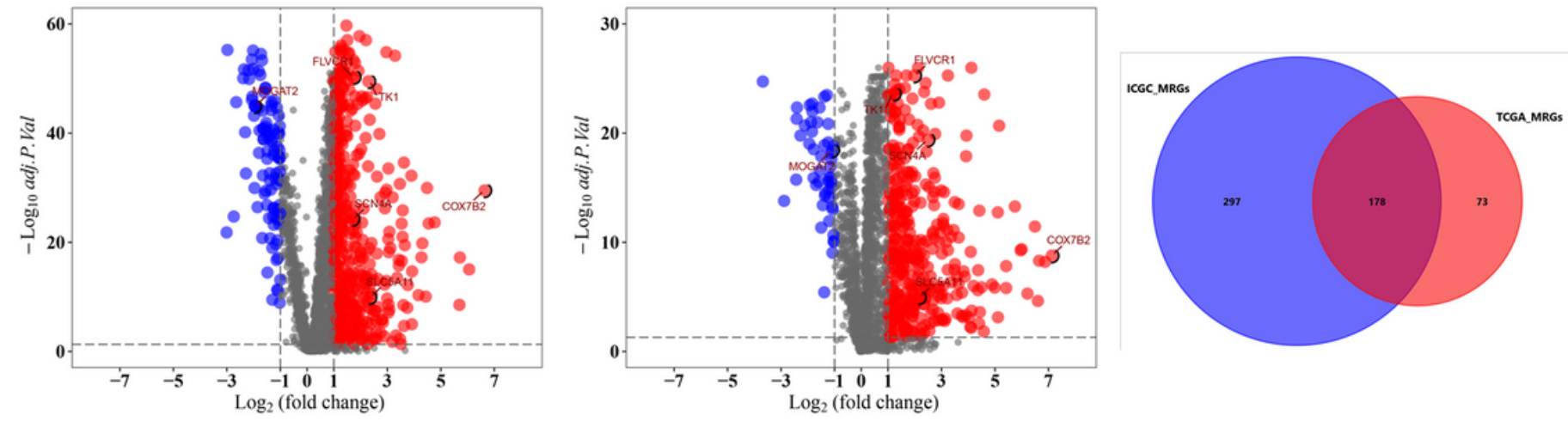

\section{Figure 1}

Differentially expressed metabolism-related genes (MRGs) in hepatocellular carcinoma (HCC). Heatmap of MRGs between HCC and nontumor tissues in ICGC database(a) and TCGA database (b). The color from blue to red represents the progression from low expression to high expression; Volcano plot of MRGs in ICGC database(c) and TCGA database(d). The red dots in the plot represents upregulated genes and blue dots represents downregulated genes with statistical significance. Black dots represent no differentially expressed genes. (e) Venn diagram showing the gene numbers of the MRGs of HCC in ICGC and TCGA database. 
Fig.2

a
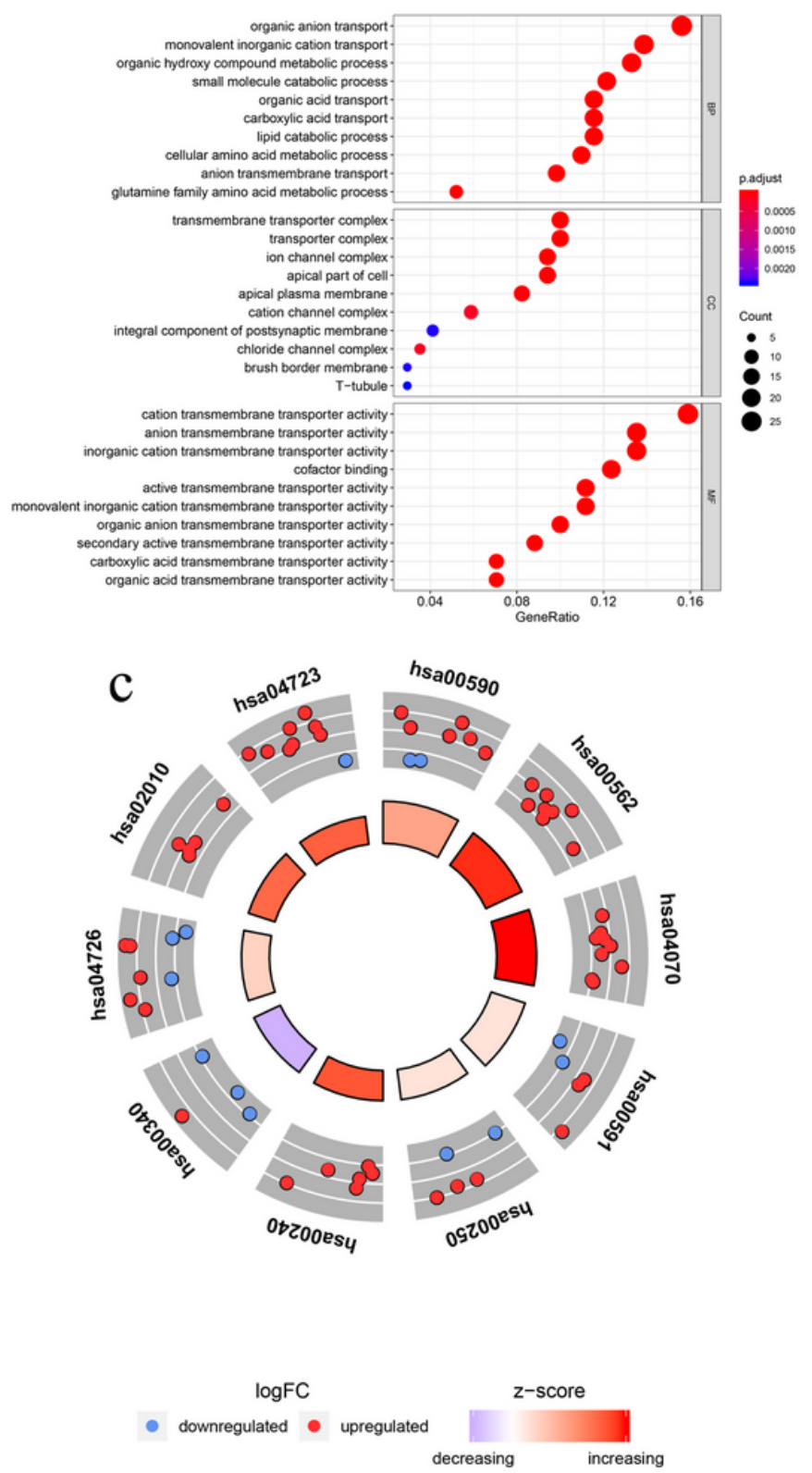

$\mathrm{b}$

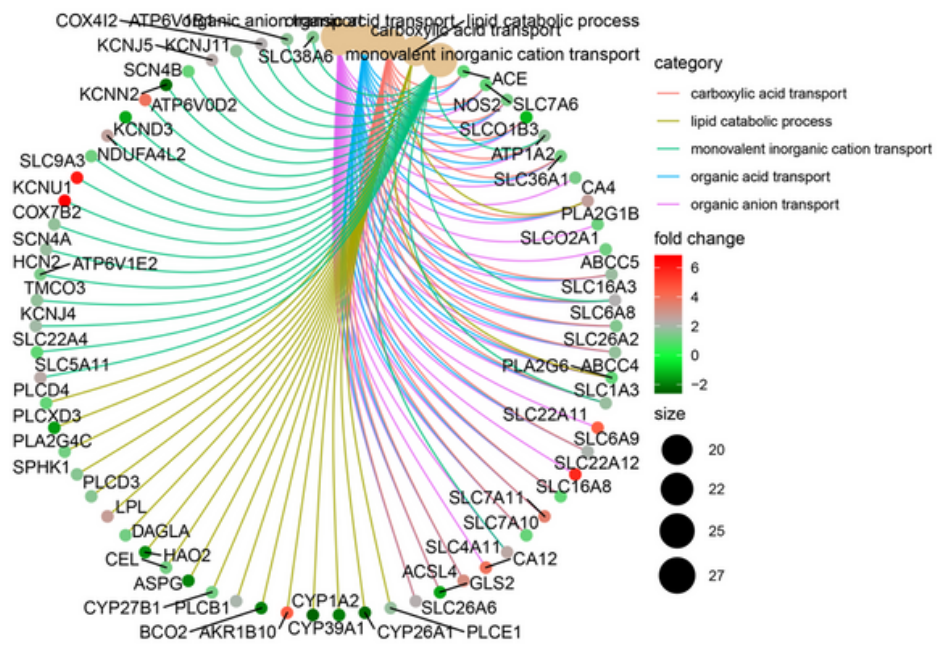

\begin{tabular}{|r|r|}
\hline ID & Description \\
\hline hsa00590 & Arachidonic acid metabolism \\
\hline hsa00562 & Inositol phosphate metabolism \\
\hline hsa04070 & Phosphatidylinositol signaling system \\
\hline hsa00591 & Linoleic acid metabolism \\
\hline hsa00250 & Alanine, aspartate and glutamate metabolism \\
\hline hsa00240 & Pyrimidine metabolism \\
\hline hsa00340 & Histidine metabolism \\
\hline hsa04726 & Serotonergic synapse \\
\hline hsa02010 & ABC transporters \\
\hline hsa04723 & Retrograde endocannabinoid signaling \\
\hline
\end{tabular}

Figure 2

The GO and KEGG analysis of differentially expressed MRGs. (a) the top 10 of biological processes GO terms, cellular component GO terms, molecular function GO terms; (b) The correlation between intersection genes and top 5 biological processes GO terms; (c) The KEGG pathway analysis of differentially expressed MRGs; 


\section{Fig.3}

a

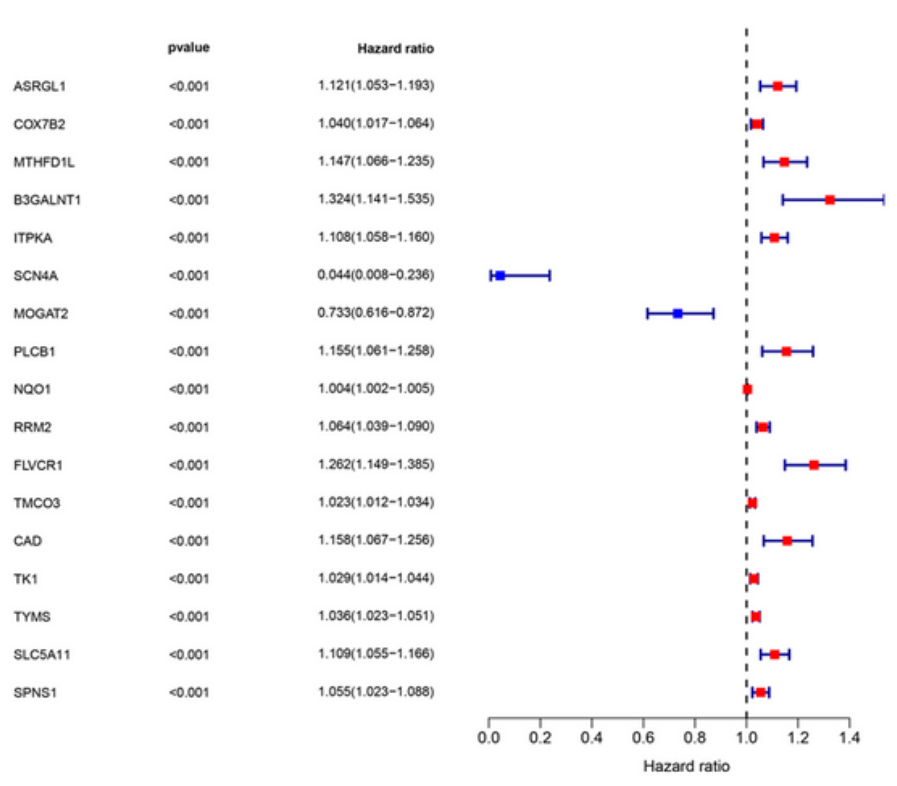

b

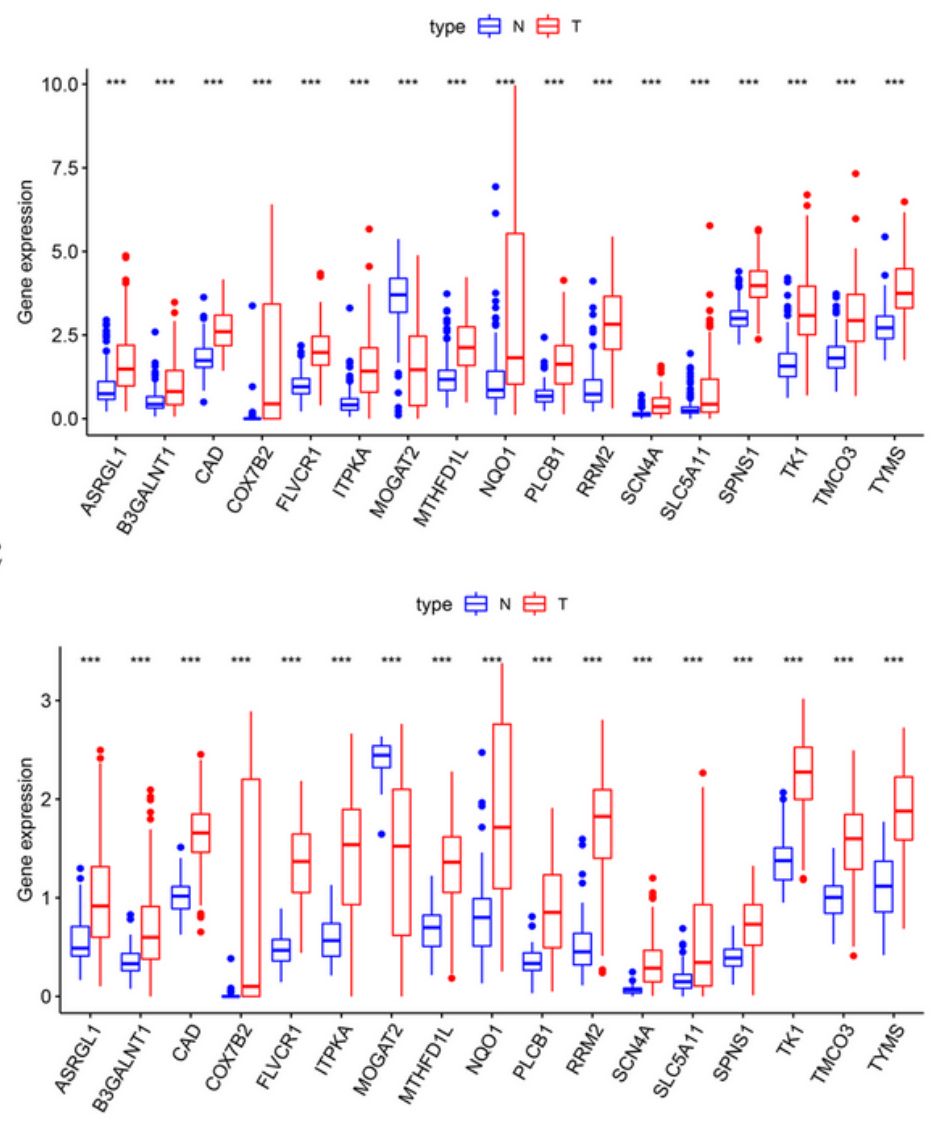

\section{Figure 3}

Identification of survival-related differentially expressed MRGs by univariate Cox regression analysis. (a) Forest plot of hazard ratios showing survival-related MRGs. P values $<0.001$ are considered to be statistically significant; The expression of 17 metabolism-related prognostic genes between HCC and normal tissues in ICGC database(b) and TCGA database(c). 
Fig. 4
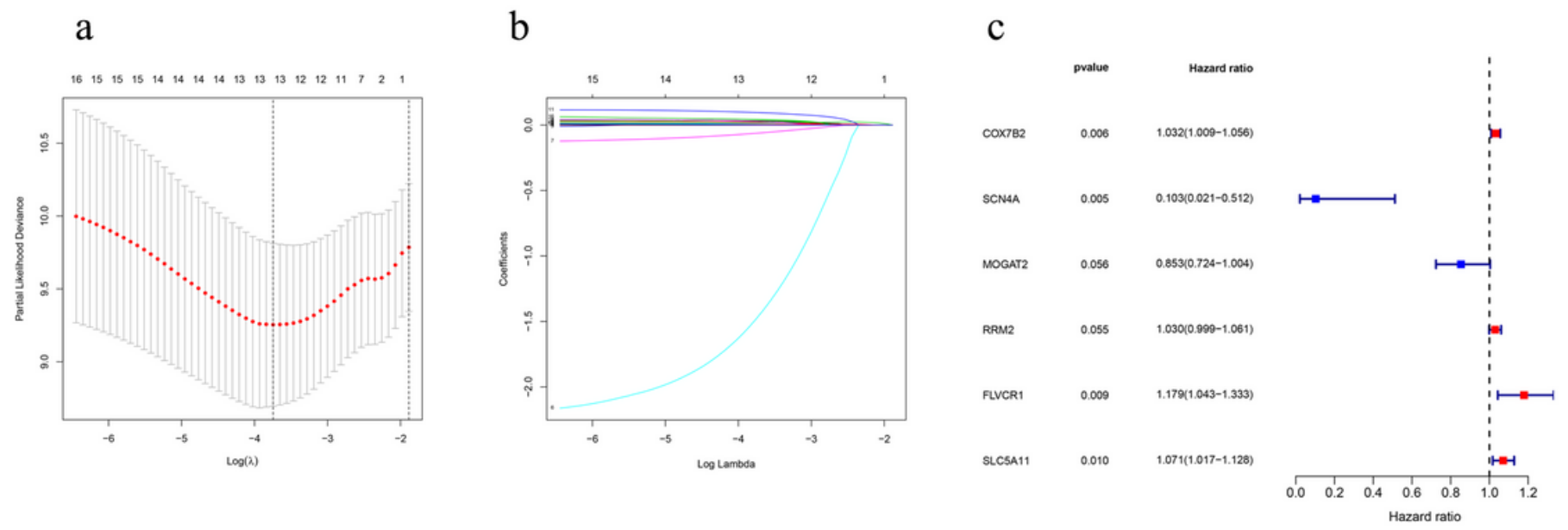

Figure 4

Establishment of metabolism-related prognostic signature. (a)Screening of optimal parameter (lambda) at which the vertical lines were drawn. (b)Lasso coefficient profiles of the seventeen MRGs with non-zero coefficients determined by the optimal lambda. (c)Multivariate analyses assessing relationship between expression levels of MRGs and overall survival (OS) in patients with HCC. 
Fig.5

a

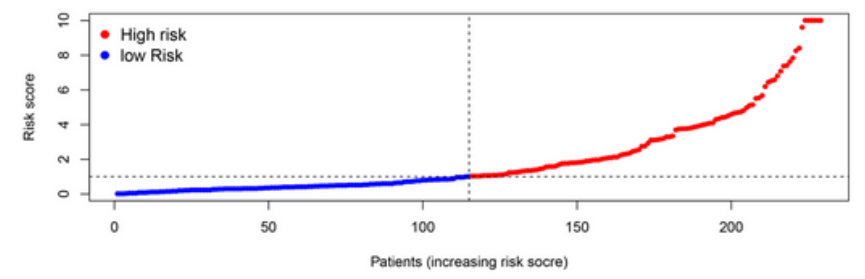

b

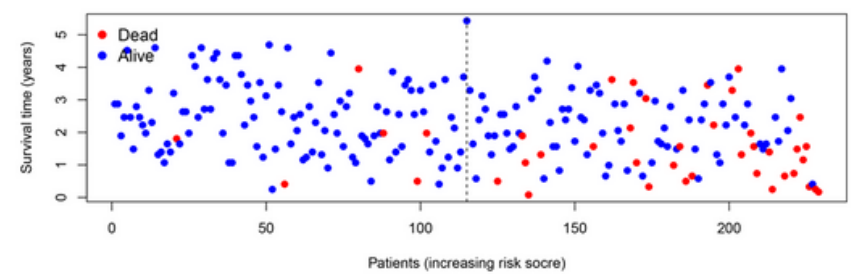

c

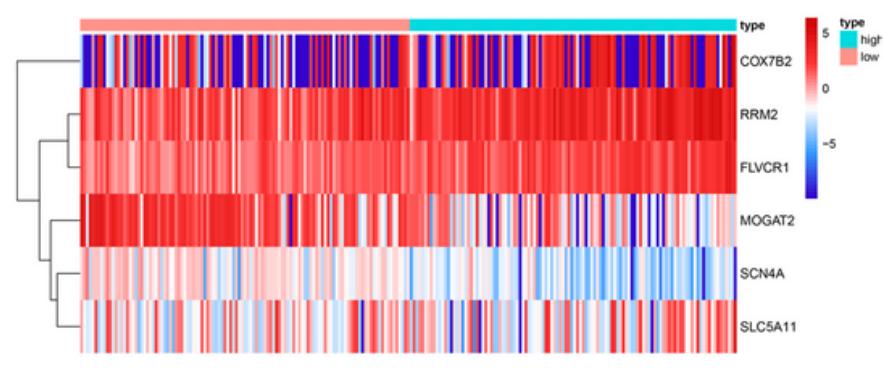

d
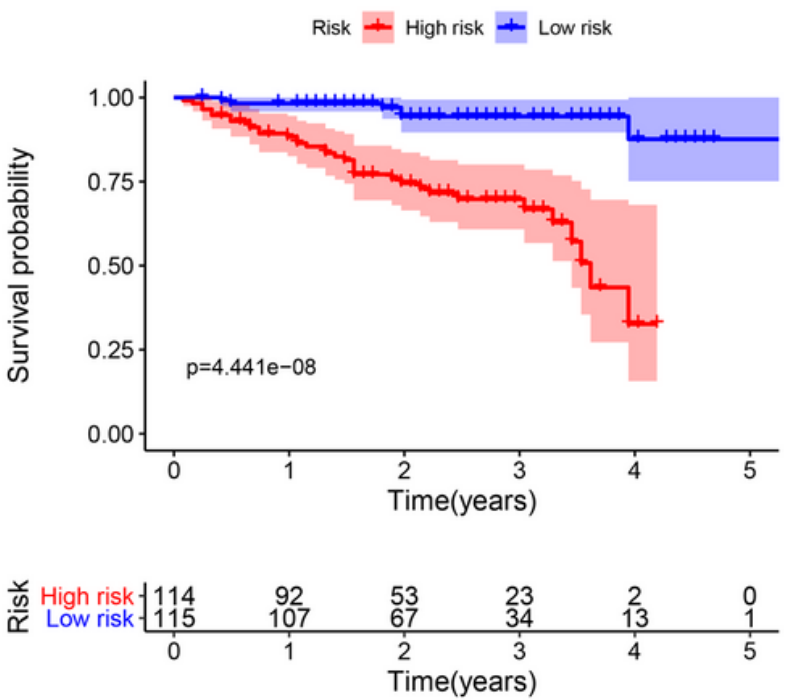

e

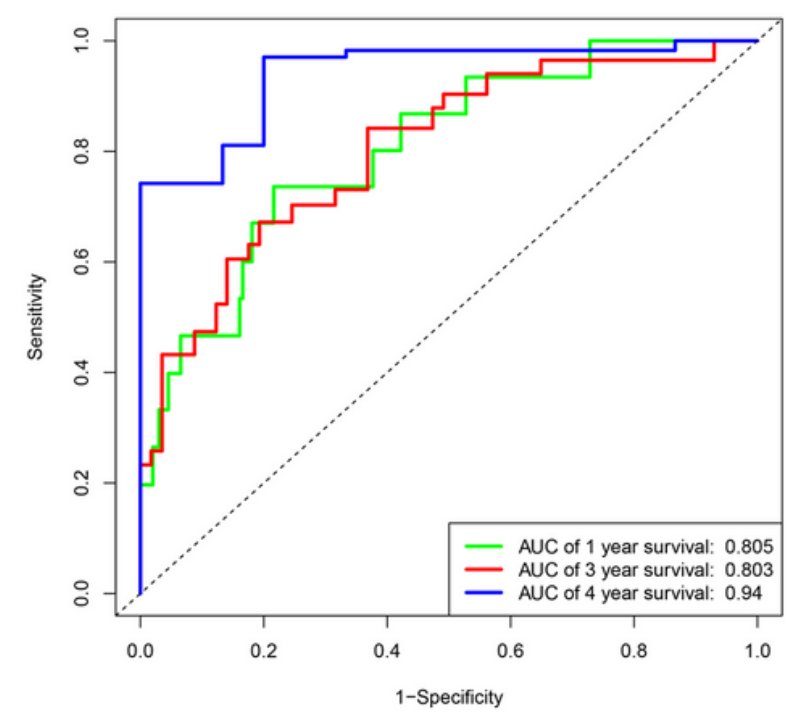

Figure 5

Construction of the metabolism-based prognostic risk signature in the ICGC cohorts. (a)The risk score distribution of HCC patients; (b)Survival status and duration of patients;(c)Heatmap of the metabolismrelated genes expression; (d)Survival curves for the low risk and high risk groups; (e)Time-independent receiver operating characteristic (ROC) analysis of risk scores for prediction the overall survival in the ICGC set. 
Fig. 6

a

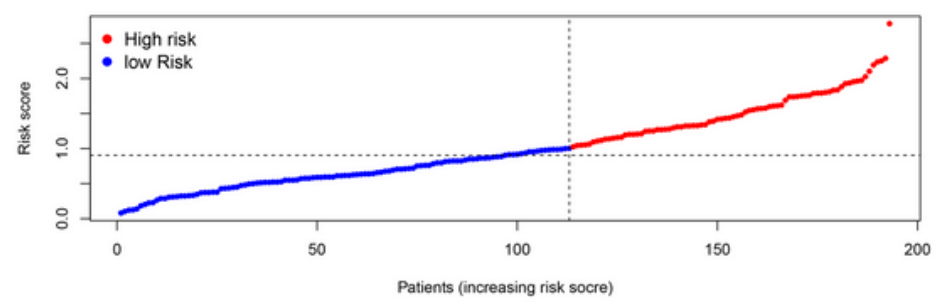

b

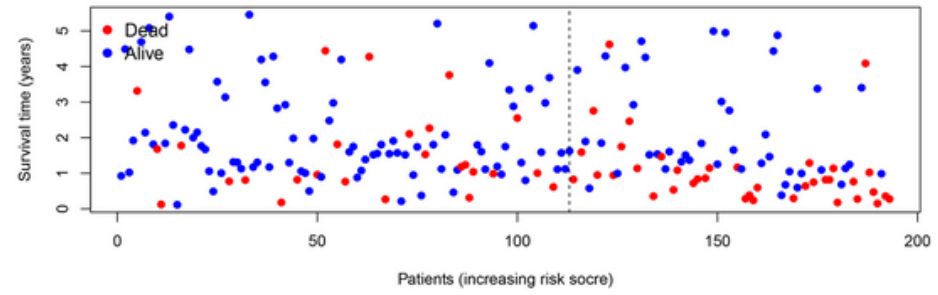

c

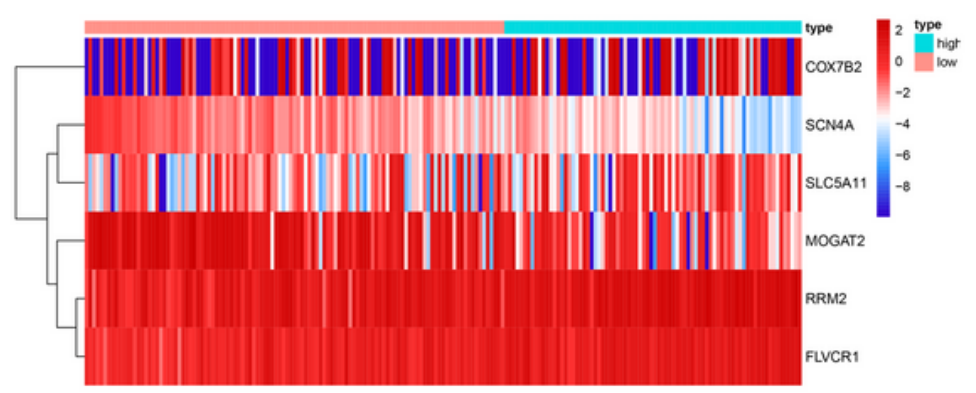

f

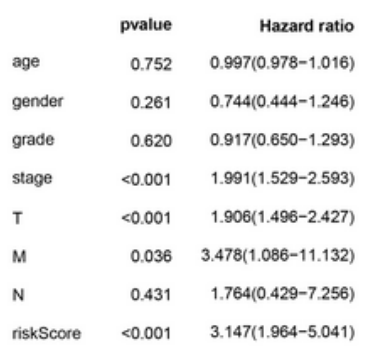

d
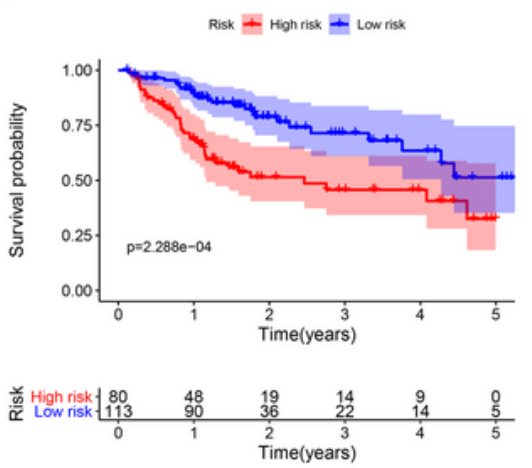

e

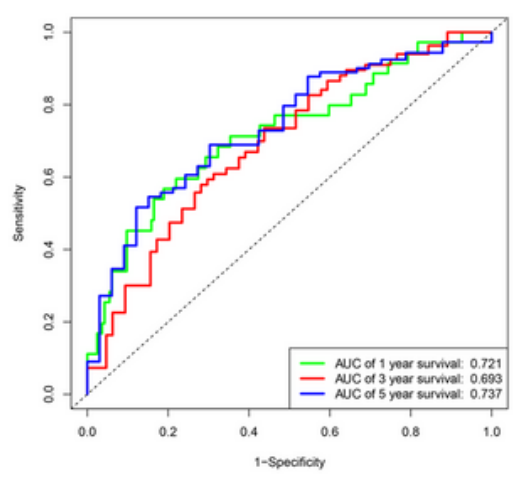

\section{Figure 6}

Validation of the metabolism-based prognostic risk signature in the TCGA cohorts. (a)The risk score distribution of HCC patients; (b)Survival status and duration of patients;(c)Heatmap of the metabolismrelated genes expression; (d)Survival curves for the low risk and high risk groups; (e)Time-independent receiver operating characteristic (ROC) analysis of risk scores for prediction the overall survival in the 
TCGA set. (f) Univariate Cox regression analysis of discrete clinical factors ; (g) Multivariate Cox regression analysis of discrete clinical factors.

\section{Fig. 7}

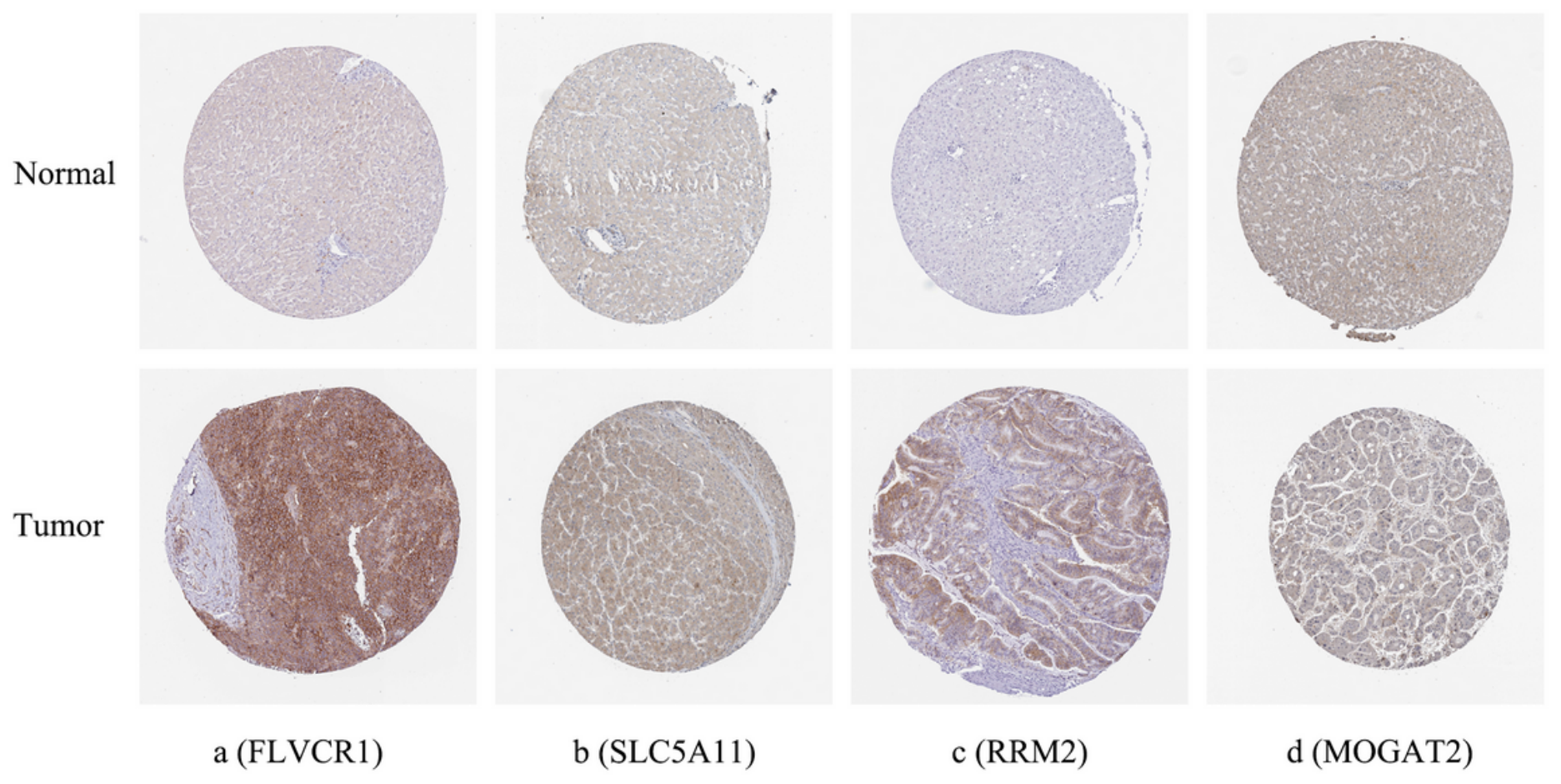

\section{Figure 7}

Verification of hub MRGs expression in HCC and normal liver tissue using the HPA database. (a): FLVCR1, (b):SLC5A11, (c): RRM2, (d): MOGAT2. 
Fig. 8
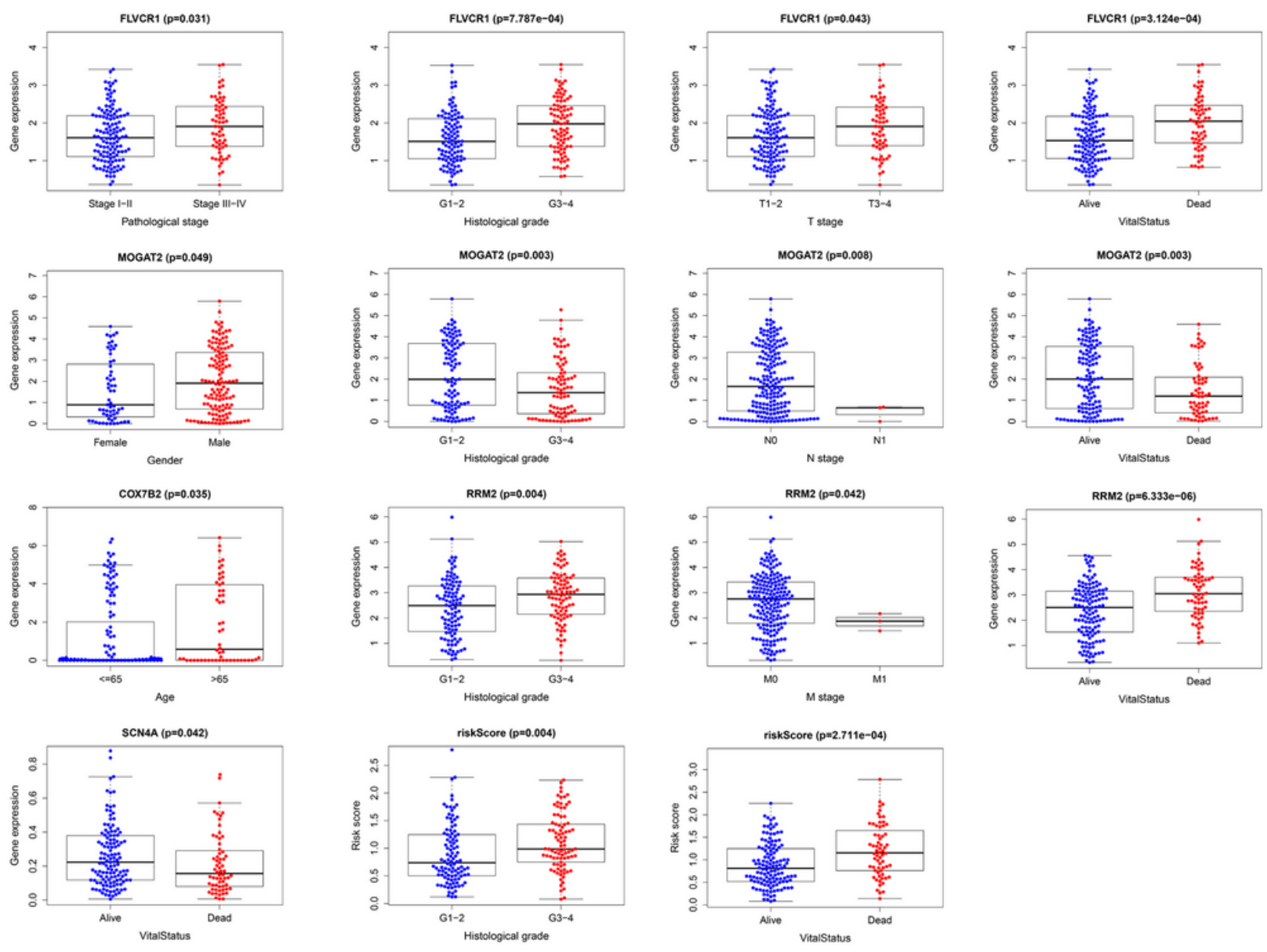

\section{Figure 8}

Relationships between MRGs expression and clinicopathological factors in HCC $(P<0.05)$. 
Fig.9

A
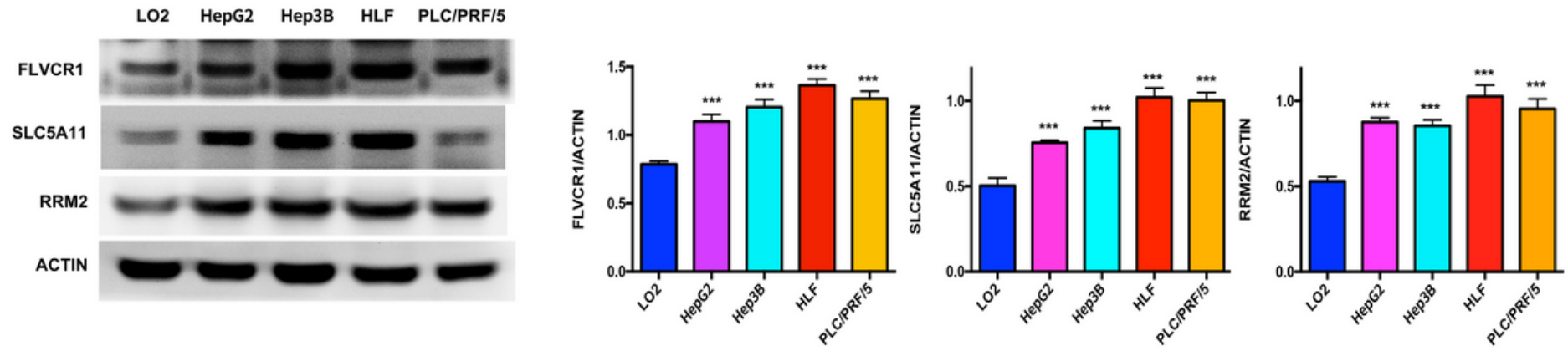

B
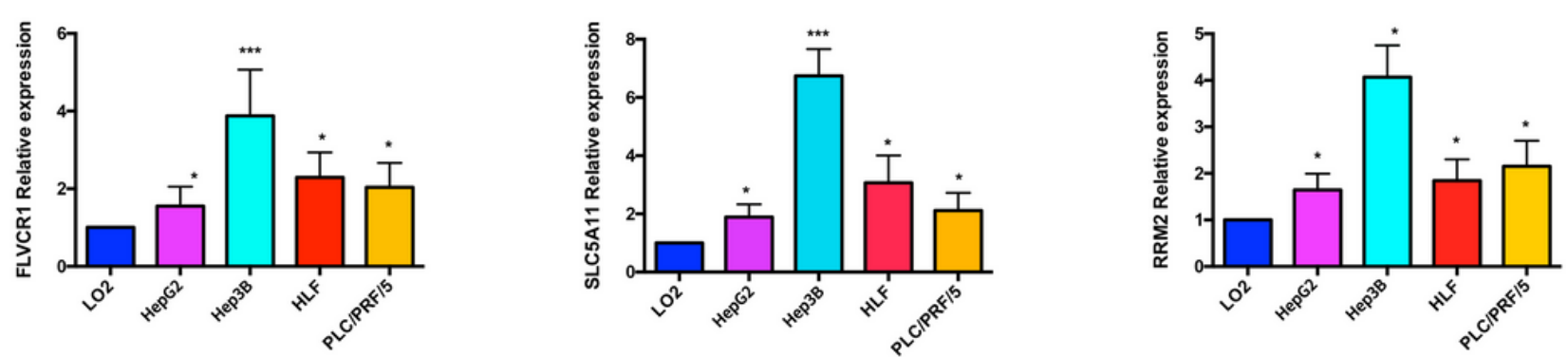

Figure 9

Validation of hub genes by WB and qRT-PCR. WB(a) and qRT-PCR(b) validation of the expression of FLVCR1, SLC5A11 and RRM2 in cell lines. 